\title{
Inappropriate weaning and late onset ventilatory failure of individuals with traumatic spinal cord injury*
}

\author{
J R Bach MD FCCP FAAPMR
}

Associate Professor and Vice Chairman, Department of Physical Medicine and Rehabilitation, University of Medicine and Dentistry of New Jersey, New Jersey Medical School, University Hospital B-239, 150 Bergen Street, Newark, New Jersey, USA.

\begin{abstract}
Eight traumatic spinal cord injured (SCI) individuals are described. Four tetraplegic patients who were weaned from initial ventilator use despite severe restrictive pulmonary syndromes experienced multiple pulmonary complications, episodes of acute respiratory failure, and 3 of the 4 required long term ventilatory support within 2 years of initial weaning. Three other SCI individuals developed chronic late-onset ventilatory failure 17, 25, and 29 years postinjury. One other tetraplegic patient was mechanically ventilated for 8.2 years postinjury despite complete recovery of autonomous ventilatory function years earlier. Of the 6 patients requiring long term ventilatory support, 4 were managed by noninvasive techniques of intermittent positive pressure ventilation (IPPV), one by negative pressure body ventilators, and one by tracheostomy IPPV. At least one and possibly as many as 5 patients were spared bronchoscopy and/or pulmonary complications by using mechanical insufflation-exsufflation (MI-E). We conclude that patients who are weaned despite having significant restrictive pulmonary syndromes are at risk for ventilatory decompensation weeks to decades after weaning. Ongoing clinical assessment, evaluation of respiratory muscle function and, at times, nocturnal blood gas analyses are warranted in the follow up of traumatic SCI individuals.
\end{abstract}

Keywords: spinal cord injury; quadriplegia; respiratory paralysis; mechanical ventilation.

\section{Introduction}

Inspiratory muscle weakness can lead to chronic alveolar hypoventilation $(\mathrm{CAH})$. The risk of pulmonary morbidity and mortality correlates with the extent of hypercapnia, ${ }^{1,2}$ and, at least for individuals with restrictive pulmonary conditions, with difficulty in clearing airway secretions from inadequate peak cough expiratory flows (PCEFs) due to expiratory muscle weakness. ${ }^{3}$ For neuromuscular patients awake hypercapnia generally develops when the vital capacity (VC) sitting decreases to $40 \%^{4}$ to $55 \%^{5}$ of predicted normal.

*This work was performed at University Hospital, Newark, NJ, USA.
However, CAH is often first noted as hypercapnia during sleep. ${ }^{6}$ When ventilation becomes normal by the use of respiratory muscle assistance including ventilatory support, blood gases improve, the risk of pulmonary complications decreases, and survival is prolonged, ${ }^{7-13}$ with the greatest benefit for patients without significant concomitant lung disease.

In the general population the VC plateaus at age 19 and then decreases at a rate of $1 \%$ to $1.2 \%$ or about $30 \mathrm{ml}$ per year. ${ }^{14}$ The VCs of individuals with progressive neuromuscular conditions can plateau during childhood and then decrease rapidly. ${ }^{15,16}$ The VC also decreases during acute paralytic poliomyelitis. It recovers to varying degrees, plateaus, then decreases once 
again by as much as $1.7 \%{ }^{17}$ to $1.9 \%^{18}$ per year. $\mathrm{CAH}$ develops and, although considered a relatively stable condition, post-poliomyelitis individuals may experience numerous pulmonary complications and late-onset ventilator dependence. ${ }^{19}$ This is particularly true for individuals who used ventilators during acute poliomyelitis. In one study 71 individuals required lateonset ventilatory assistance 3 to 59 years or a mean of $29 \pm 12.1$ years post poliomyelitis. ${ }^{19}$

Ventilatory assistance is often initially required overnight and only gradually becomes necessary during daytime hours. In a recent study, neuromuscular patients required nocturnal ventilatory assistance when their supine VCs had decreased to about $30 \%$ of predicted normal. ${ }^{7}$ Although supine VC measurements are useful they are not often performed.

For patients with traumatic tetraplegia who may or may not have required ventilator use at the onset, the $\mathrm{VC}$ also plateaus at some time following injury and then decreases with age. The VCs in the sitting position of stable $\mathrm{C} 4-\mathrm{C} 7$ tetraplegics range from $42 \% \quad(\text { range }=28-63 \%)^{20}$ to $52 \pm$ $11 \%^{21}$ of predicted normal. However, supine VCs which are usually greater than sitting VCs may be significantly less than sitting VCs when the patient is adept at supplementing inspiratory volumes by accessory muscle use. With the loss of pulmonary volumes and function over time, we report for the first time that some SCI individuals are, in fact, subject to late-onset chronic ventilatory insufficiency. Likewise, patients may be inappropriately weaned from ventilator use during the initial injury recovery period or be maintained on ventilatory support longer than needed. These points, too, have not been previously reported. Basic patient data are provided in Table I.

\section{Case studies:}

Late-onset chronic ventilatory failure

Case 1, a male tetraplegic, developed a mild S-shaped thoracolumbar curve and hypersomnolence due to CAH in 1969, 12 years postinjury. He was treated for 5 years with 24-hour home oxygen therapy. However, his pCO2 increased to 70 to $80 \mathrm{mmHg}$ and the hypersomnolence worsened. His first hospital admission for respiratory failure was triggered by an upper respiratory tract infection (URI) in 1974. He was intubated, had a tracheostomy and received tracheostomy IPPV for 3 weeks before weaning and tracheostomy site closure. Subsequent complications were associated with episodes of bronchitis and/or pneumonia before beginning definitive daily ventilatory aid (Table Ib). In 1983, he developed right ventricular failure and had three respiratory arrests during attempts to wean from IPPV via a nasotracheal tube following urological surgery. He was converted to nocturnal chest shell ventilator aid which he used for 4 years. He then switched to a negative pressure wrap ventilator until 1989. From 1983 to 1989 he was hospitalised on nine occasions and intubated eight times. He had undergone bronchoscopic mucus removal on 13 occasions by this time. In 1989, with a VC of $450 \mathrm{ml}$ sitting and $580 \mathrm{ml}$ supine, arterial blood gases and continuous noninvasive blood gas monitoring demonstrated the ineffectiveness of nocturnal wrap ventilator use. Awake, unaided $\mathrm{pCO}_{2}$ ranged from $55 \mathrm{mmHg}$ to $95 \mathrm{mmHg}$ and $\mathrm{pO}_{2}$ from $40 \mathrm{mmHg}$ to $50 \mathrm{mmHg}$ on room air. The results of the nocturnal $\mathrm{SaO}_{2}$ monitoring are given in Table II. He was switched to nocturnal mouthpiece IPPV but refused to use a Bennett lipseal (Puritan-Bennett, Boulder, $\mathrm{CO}$ ), which is recommended for overnight use, ${ }^{8}$ because of the inability to independently remove the retention straps to use a sip-and-puff operated telephone during the night. His blood gases were not significantly improved by using nocturnal mouthpiece IPPV without a lipseal. Since he retained fair plus strength of the right biceps a pulley system was devised which allowed him to use a lipseal and remove it independently (Fig 1). Lipseal IPPV resulted in normalization of nocturnal $\mathrm{SaO}_{2}$ and alveolar ventilation with the latter monitored by end-tidal $\mathrm{CO}_{2}$ measurements. Oximetry and occasionally capnography biofeedback demonstrated normalization of $\mathrm{SaO}_{2}$ and alveolar ventilation during daytime mouthpiece IPPV. Mouthpiece IPPV also relieved 
Table Ia Clinical data

\begin{tabular}{lccccc}
\hline DOB $^{\mathrm{a}}$ & Injury date & Level & $\begin{array}{c}\text { Initial } \\
\text { support }\end{array}$ & $\begin{array}{c}\text { VC sittingc } \\
\text { (ml/\% pred) }\end{array}$ & $\begin{array}{c}\text { VC supinec }^{\mathrm{c}} \\
\text { (ml/\% pred) }\end{array}$ \\
\hline $7 / 44$ & $6 / 57$ & $\mathrm{C} 4-5$ & no & $450 / 14 \%$ & $520 / 16 \%$ \\
$6 / 49$ & $6 / 49$ & T5 & no & $730 / 25 \%$ & $585 / 20 \%$ \\
$3 / 46$ & $8 / 68$ & $\mathrm{C} 3-4$ & yes & $870 / 24 \%$ & $970 / 27 \%$ \\
$4 / 58$ & $5 / 70$ & $\mathrm{C} 4$ & yes & $670 / 18 \%$ & $700 / 19 \%$ \\
$3 / 40$ & $8 / 76$ & $\mathrm{C} 4-5$ & yes & $720 / 25 \%$ & $800 / 28 \%$ \\
$7 / 51$ & $1 / 81$ & $\mathrm{C} 4$ & yes & $450 / 13 \%$ & $-700 \%$ \\
$3 / 25$ & $7 / 92$ & $\mathrm{C} 4-5$ & yes & $900 / 30 \%$ & $900 / 30 \%$ \\
$6 / 63$ & $11 / 79$ & $\mathrm{C} 4-5$ & yes & $2920 / 68 \%$ & $3170 / 74 \%$ \\
\hline
\end{tabular}

${ }^{a}$ Date of birth; ${ }^{b}$ need for ventilatory support in the acute injury period; ${ }^{c}$ vital capacity when initially weaned from ventilator use only the maximum observed VC in at least five attempts is recorded (Wright spirometer, Mark 14, Ferraris Development and Engineering CO, Ltd, London).

Table Ib Pulmonary complications from initial weaning to the second long term period of ventilator use

\begin{tabular}{|c|c|c|c|c|c|c|c|}
\hline \multicolumn{4}{|c|}{ Before definitive aid } & \multicolumn{2}{|c|}{ Definitive support } & \multicolumn{2}{|c|}{ Type of support ${ }^{d}$} \\
\hline \multicolumn{3}{|c|}{ Times requiring ${ }^{\mathrm{a}}$} & \multirow[b]{2}{*}{ Years $^{b}$} & \multirow[b]{2}{*}{ Nocturnal } & \multirow[b]{2}{*}{$>14$ hours $^{\mathrm{c}}$} & \multirow[b]{2}{*}{ Nocturnal } & \multirow[b]{2}{*}{ Daytime } \\
\hline Hosp & Support & Intub & & & & & \\
\hline 10 & 6 & 5 & 9 & $7 / 83^{e}$ & $4 / 89$ & MIPPV & MIPPV \\
\hline 2 & 2 & 0 & 3 & $4 / 78$ & - & MIPPV & MIPPVg \\
\hline 4 & 3 & 3 & 2 & $8 / 87^{f}$ & $1 / 91$ & TIPPV & TIPPV \\
\hline 3 & 3 & 1 & 2 & $6 / 72$ & - & MIPPV & MIPPVg \\
\hline 1 & 1 & 0 & 1 & $7 / 77$ & - & IL & MIPPVg \\
\hline 1 & 1 & 0 & 0.4 & $9 / 81$ & - & MIPPV & MIPPVg \\
\hline 3 & 1 & 1 & 0.3 & $12 / 92$ & - & NIPPV & MI-E \\
\hline- & - & - & - & \multicolumn{4}{|c|}{ no second episode of ventilator use } \\
\hline
\end{tabular}

${ }^{\mathrm{a}}$ Hosp, hospitalizations. Support, number of times requiring short periods of ventilatory support by either tracheal intubation or the use of negative pressure ventilators. Intub, oro- or nasotracheal intubation for intermittent positive pressure ventilatory support (IPPV).

${ }^{b}$ Years from the first post-acute injury hospitalization for pulmonary complications to the onset of definitive daily ventilatory support.

c Use of greater than 14 hours of daily ventilatory support.

${ }^{\mathrm{d}}$ MIPPV, IPPV via mouthpiece; TIPPV, IPPV via indwelling tracheostomy tube; IL, iron lung; MI-E, mechanical insufflation-exsufflation.

eUsed nocturnal negative pressure ventilatory assistance until switched to nearly 24 hour/day MIPPV in $4 / 89$.

f Used nocturnal nasal IPPV from 8/87 until an indwelling tracheostomy was placed on 1/91.

gDaytime mouthpiece IPPV used during respiratory tract infections.

${ }^{h}$ Mechanical insufflation-exsufflation was used on a daily basis to remove airway secretions and for periodic hyperinsufflation.

daytime fatigue and dyspnea. ${ }^{22,23}$ His VC increased to $720 \mathrm{ml}$ sitting and $760 \mathrm{ml}$ supine within 4 months. His need of mouthpiece IPPV to maintain normal $\mathrm{SaO}_{2}$ decreased from 24 hours to, currently, 14 hours per day. He has had neither respiratory complications nor hospitalizations over the last 4 years and has relied on mechanical insufflation-exsufflation (MI-E) (The In-exsufflator, J H Emerson Company, Cambridge, 
Table II Results of nocturnal oxyhemoglobin saturation monitoring for case 1 using noninvasive ventilatory support

\begin{tabular}{lclrlr}
\hline Wrap ventilator use & & MIPPV without LS & & \multicolumn{2}{l}{ MIPPV with LS } \\
\hline 16 hr mean $\mathrm{SaO}_{2}{ }^{\mathrm{c}}$ & $89 \%$ & $71 \mathrm{hr}$ mean $\mathrm{SaO}_{2}$ & $89 \%$ & $118 \mathrm{hr}$ mean $\mathrm{SaO}_{2}$ & $98 \%$ \\
$\%$ time $\mathrm{SaO}_{2}<90 \%$ & $30 \%$ & $\%$ time $\mathrm{SaO}_{2}<90 \%$ & $54 \%$ & $\%$ time $\mathrm{SaO}_{2}<90 \%$ & $0 \%$ \\
$\%$ time $\mathrm{SaO}_{2}<85 \%$ & $12 \%$ & $\%$ time $\mathrm{SaO}_{2}<85 \%$ & $21 \%$ & $\%$ time $\mathrm{SaO}_{2}<85 \%$ & $0 \%$ \\
$\%$ time $\mathrm{SaO}_{2}<80 \%$ & $7 \%$ & $\%$ time $\mathrm{SaO}_{2}<80 \%$ & $6 \%$ & $\%$ time $\mathrm{SaO}_{2}<80 \%$ & $0 \%$ \\
$\%$ time $\mathrm{SaO}_{2}<70 \%$ & $2 \%$ & $\%$ time $\mathrm{SaO}_{2}<70 \%$ & $1 \%$ & $\%$ time $\mathrm{SaO}_{2}<70 \%$ & $0 \%$ \\
low $\mathrm{SaO}_{2}$ & $47 \%$ & low $\mathrm{SaO}_{2}$ & $52 \%$ & low $\mathrm{SaO}_{2}$ & $89 \%$ \\
$\mathrm{dSaO}_{2}>3 \% / \mathrm{hr}^{\mathrm{d}}$ & 28 & $\mathrm{dSaO}_{2}>3 \% / \mathrm{hr}$ & 17 & $\mathrm{dSaO}_{2}>3 \% / \mathrm{hr}$ & 1 \\
\hline
\end{tabular}

${ }^{a}$ Mouthpiece IPPV without Bennett lipseal retention.

${ }^{b}$ Mouthpiece IPPV with Bennett lipseal retention.

${ }^{\mathrm{c}} \mathrm{SaO}_{2}$, oxyhemoglobin saturation.

${ }^{\mathrm{d}} \mathrm{dSaO}_{2}>3 \%$, oxyhemoglobin desaturations of greater than $3 \%$ per hour.

a

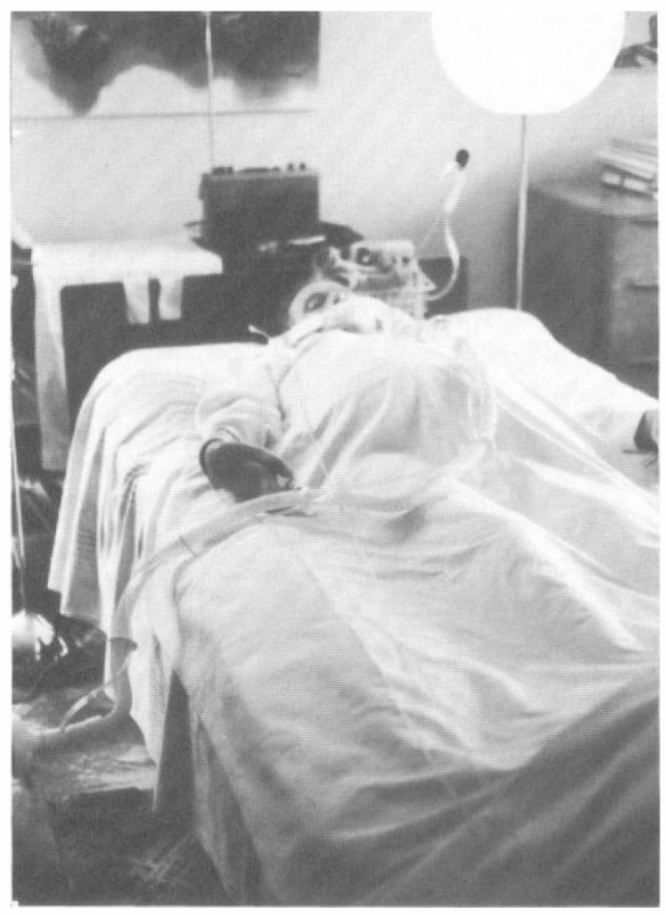

MA) to clear airway secretions during URIs. ${ }^{24,25}$ On December 22,1992 his VC was $680 \mathrm{ml}$ sitting and $720 \mathrm{ml}$ supine. His maximum PCEFs measured from inspiratory capacity were $0.9 \mathrm{l} / \mathrm{s}$ unassisted, $1.2 \mathrm{l} / \mathrm{s}$ with an abdominal thrust following a maximum insufflation of $1100 \mathrm{ml}$, and $6.9 \mathrm{l} / \mathrm{s}$ by MI-E. 25

Case 2, a female, complained of shortness of breath, fatigue, difficulty clearing bron- $\mathbf{b}$

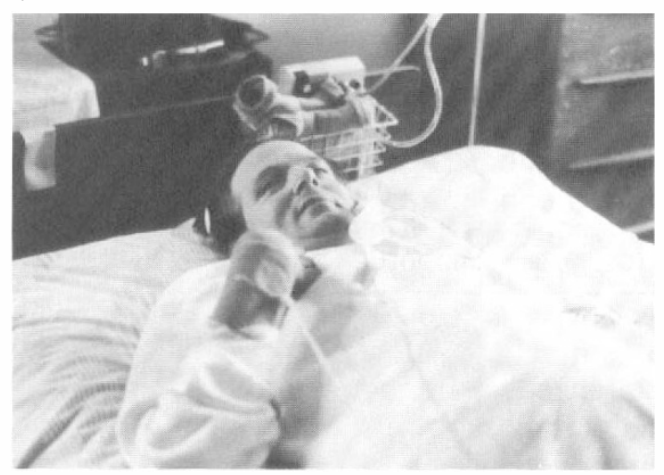

Figure 1 Traumatic quadriplegic patient with late-onset chronic ventilatory insufficiency using 14 to 24 hour a day mouthpiece intermittent positive pressure ventilation. He has fair plus strength of his right biceps with which he can operate a pulley system to release his lipseal during overnight use. (a) The cord attached to the lipseal extends to a pulley at the foot of the bed then to a wrist band. (b) Flexion of the arm releases the lipseal.

chial secretions, frequent awakening from sleep with tachycardia and the sensation of suffocating, orthopnea, and depression in 1978, 29 years following a T5 spinal injury at birth. She could generate only short, faintly spoken sentences. There was severe edema, right ventricular insufficiency, and moderate scoliosis. Her VC was $730 \mathrm{ml}$ sitting. Oxygen therapy did not relieve the symptoms. After one episode of pneumonia, and 
severe hypercapnia which resulted in two respiratory arrests, hospitalization, and iron lung use, she was placed on nocturnal lipseal IPPV. ${ }^{8}$ Supplemental oxygen was discontinued. Her symptoms were completely relieved and daytime blood gases improved. She has had neither pulmonary complications nor hospital readmission over the past 15 years. Sleep oximetry on lipseal IPPV supine demonstrated a mean $\mathrm{SaO}_{2}$ of $97 \%$. During URIs she requires mouthpiece IPPV 24 hours a day for adequate alveolar ventilation and to improve her cough. Her VC has decreased to $690 \mathrm{ml}$ sitting $(21 \%$ of predicted normal) and $585 \mathrm{ml}$ (16\% of predicted) supine since 1978.

Case 3 is an out-of-state male tetraplegic physician who required ventilatory support for 3 weeks following injury in 1968, with no respiratory difficulties until 1985 . From 1985 to September 1987 he was hospitalized, intubated, and weaned from ventilator use to continuous home oxygen therapy on three occasions. On the last occasion, just prior to intubation for an acute respiratory arrest, his $\mathrm{pCO}_{2}$ was noted to be $105 \mathrm{mmHg}$. With IPPV he awoke, was stabilized, then extubated and placed on nocturnal nasal IPPV in July 1987. This improved alveolar ventilation and daytime blood gases temporarily but he became severely hypercapnic when unaided. The use of daytime ventilatory aid was not arranged. Dyspnea increased and he became uncomfortable with commercial nasal interfaces. Custom interfaces were not obtained. There was also no use of effective assisted coughing. He was hospitalized for ventilatory insufficiency in August 1988, and hospitalized and intubated for several month periods in March 1989 and three times in 1990. In January of 1991 he arrested while in his hospital office, and he was intubated, resuscitated, and underwent tracheostomy which he continues to use 24 hours a day. He ceased all employment activities at that time. Laryngeal damage, presumably from intubation, has prevented effective verbal communication since January 1991 . He has had multiple episodes of mucus plugging, pneumonia, acute respiratory insufficiency and intensive care admissions since January 1991.
Inappropriate ventilator weaning

Case 4 is a tetraplegic lady who was injured at age 12 years in 1970. She received tracheostomy IPPV for 6 weeks then was weaned and the tracheostomy site was allowed to close. However, she was symptomatic for hypoventilation with fatigue and sleep arousals with dyspnea and tachycardia. She also developed pneumonia and ventilatory failure on three occasions during the first 2 years postinjury. At age 14 she was placed on nocturnal lipseal IPPV. She also used mouthpiece IPPV for daytime periods when fatigued and during URIs. Her VC plateaued at age 17 years at $760 \mathrm{ml}$ sitting. She had no further complications nor hospitalizations from 1972 until at least 1985 when she was lost to follow up. By age 27 in 1985 her VC had decreased to $670 \mathrm{ml}$ (18\%) sitting and $700 \mathrm{ml}(19 \%)$ supine.

Case 5, a 36 year old male tetraplegic patient, was injured in 1976 . He was intubated and had a tracheostomy for IPPV for 2 months, then weaned, and returned asymptomatic to the community. In July 1977 an URI developed into pneumonia and respiratory failure. Weaning was not possible without dyspnea and hypercarnia. His symptoms were relieved by nocturnal chest shell ventilator use. After one year he switched to more effective nocturnal iron lung use. He has had four URIs but no respiratory complications nor hospitalizations while using negative pressure aids. He currently has no ability to breathe autonomously when supine. $\mathrm{He}$ is also hypercapnic and dyspneic when sitting and using accessory muscles. The chronic care facility in which he resides, however, does not permit the use of positive pressure ventilators for daytime use.

Case 6 is a 29 year old male tetraplegic patient. He was intubated, used tracheostomy IPPV for 5 months, then weaned and the tracheostomy site was closed despite a VC of $450 \mathrm{ml}$ sitting and complaints of chronic fatigue. Four months later an URI developed into pneumonia. Ventilatory failure was treated by 24-hour mouthpiece IPPV. He was subsequently weaned to nocturnal lipseal IPPV. At yearly evaluations from 1981 to 1986 his sitting VCs were $450 \mathrm{ml}, 460 \mathrm{ml}, 580 \mathrm{ml}, 890 \mathrm{ml}, 910 \mathrm{ml}$, 
and $960 \mathrm{ml}$, respectively. On April 20, 1988 he had a sitting VC of $1350 \mathrm{ml}(37 \%$ of predicted) and supine VC of $800 \mathrm{ml}(20 \%$ of predicted). Nocturnal oximetry during unaided sleep yielded a mean $\mathrm{SaO}_{2}$ of $96 \%$ with occasional severe desaturations associated with chest wall spasticity. Mouthpiece IPPV was discontinued without subsequent complications and his VC sitting increased to $2300 \mathrm{ml}$ in June 1992.

Case 7 is a male tetraplegic patient with a 29 year history of two packs/day of cigarette use. He was weaned from ventilator use 2 months postinjury. The tracheostomy tube was capped and he was given supplemental oxygen. Three days after rehabilitation admission, with profuse secretions, he developed pneumonia, became ventilator dependent for 3 weeks, then was once again weaned to oxygen therapy. At this time his tracheostomy site was allowed to close but secretions remained profuse and purulent. Two episodes of respiratory failure necessitated bronchoscopic removal of mucus plugs over the next 2 months. He was then referred to a rehabilitation ventilator unit. His VC was $900 \mathrm{ml}$, oxygen therapy was discontinued and $\mathrm{SaO}_{2}$ varied from $93 \%$ to $80 \%$ with end-tidal $\mathrm{pCO}_{2}$ to greater than $50 \mathrm{mmHg}$. He was placed on mouthpiece IPPV with oximetry biofeedback and nocturnal nasal IPPV to normalize ventilation and provide deep breaths for assisted coughing. ${ }^{22}$ His maximum unassisted PCEFs were $1.2 \mathrm{l} / \mathrm{s}$. He had frequent sudden oxyhemoglobin desaturations below $80 \%$ which were corrected with the elimination of profuse secretions and mucus plugs by MI-E coordinated with manually applied abdominal thrusts. Immediately following the initial use of MI-E his VC rose to $1200 \mathrm{ml}$. He required MI-E every 15 minutes during daytime hours for 2 days at which point secretions diminished, $\mathrm{SaO}_{2}$ remained over $94 \%$, VC increased to $1600 \mathrm{ml}$ and he discontinued IPPV. He was able to complete his rehabilitation program without further incident.

Inappropriate continuation of ventilator use Case 8 , a 24 year old male tetraplegic patient, used 24 hour/day tracheostomy IPPV for 6 months. At that point he developed a maximum of 1 hour of ventilator-free time with a VC of $340 \mathrm{ml}$. Ventilator-free time gradually increased and he was weaned to overnight IPPV over the next 4 months. He was discharged home and instructed to 'never stop using ventilatory support'. He suffered two lung collapses and pneumonia 3 years postinjury but had no other difficulties and underwent no further pulmonary function testing until presenting to us 8.2 years postinjury. At that time his VC was $2920 \mathrm{ml}$ sitting and $3170 \mathrm{ml}$ supine. Mean nocturnal $\mathrm{SaO}_{2}$ was $96 \%$ with a low of $91 \%$. IPPV was discontinued and the tracheostomy site closed. His VC increased to $4200 \mathrm{ml}$ sitting and $3600 \mathrm{ml}$ supine at 13 years postinjury.

\section{Discussion}

Four of the 6 tetraplegic patients who were ventilator weaned following acute injury developed the need for long term ventilator use. Two other patients developed lateonset need for long term ventilator use without previous need in the postinjury period. As with post-poliomyelitis patients, ventilator use at disease onset and the presence of back deformity both appear to be risk factors for late-onset chronic ventilatory insufficiency and justify close surveillance of inspiratory and expiratory muscle function. The former can be conveniently monitored by VC and/or maximum inspiratory pressure measurements. Nocturnal $\mathrm{SaO}_{2}$ monitoring should be performed whenever there are symptoms suggestive of $\mathrm{CAH}$ or sleep disordered breathing, daytime blood gases are abnormal, or if the supine VC decreases below $30 \%$ to $40 \%$ of predicted normal. Practical expiratory muscle function can be conveniently evaluated by measuring PCEFs with a simple peak flow meter. ${ }^{22}$

If untreated $\mathrm{CAH}$ can lead to pulmonary complications and acute respiratory failure. Acute failure is often triggered by an intercurrent URI during which increased expiratory muscle weakness, profuse airway secretions, and impaired secretion clearance alter pulmonary ventilation/perfusion and increase the work of breathing. Although 
none of these patients were obese nor had complicating medical conditions and only 2 had back deformity, all except cases 7 and 8 required ongoing ventilatory support and the need for 24-hour ventilatory aid during URIs.

Cases 1, 2 and 3 demonstrated that late-onset ventilatory insufficiency could develop insidiously in SCI patients. These patients had complications and repeated hospitalizations despite oxygen therapy but were successfully treated by 8 to 24 hour noninvasive IPPV. Once adequate alveolar ventilation was achieved the risk of further respiratory complications was greatly diminished. Oximetry biofeedback, manually assisted coughing and MI-E were also vital during intercurrent URIs and facilitated management in the home. The successful use of these techniques has also been described for other patient populations. ${ }^{8,19,23,25}$

In addition, case 1 demonstrated that nocturnal ventilatory aid alone may not be adequate indefinitely, and that the use of negative pressure body ventilators may be associated with severe, presumably obstructive sleep apneas. ${ }^{26}$ The use of mouthpiece IPPV during sleep without a lipseal may not provide normal alveolar ventilation. The apparent improvement in central ventilatory drive achieved by correcting alveolar ventilation during daytime hours may also improve the effectiveness of nocturnal mouthpiece IPPV. ${ }^{8}$ Similar observations have been made for patients using nocturnal nasal IPPV. ${ }^{7}$ Case 1 also demonstrated that with effective ventilatory assistance and relief of accessory muscle use and fatigue, VC and ventilator-free time can at least temporarily increase despite eventual diminution of VC with aging. Further, this patient's severely diminished unassisted PCEFs caused a high risk of pulmonary complications during URIs and following general anesthesia. PCEFs were not significantly increased by manually assisted coughing because of poor pulmonary compliance but were adequate with MI-E.

Both cases 1 and 2 demonstrated that right ventricular insufficiency could be reversed by using noninvasive ventilatory assistance. Case 2 also demonstrated the need for monitoring supine $\mathrm{VC}$. Adequate blood gases and $\mathrm{VC}$ while awake and sitting using accessory inspiratory muscles can obfuscate the presence of severe nocturnal hypoventilation. . $^{7,19}$

Case 3 demonstrated that failure to assist inspiratory muscle function in a timely manner, and the use of supplemental oxygen, can lead to severe hypercapnia and respiratory arrest. Further, nocturnal noninvasive IPPV alone will not be adequate for individuals with inadequate ventilatory function during waking hours. Custom molded interfaces should be obtained for those who are uncomfortable with generic continuous positive airway pressure (CPAP) masks, ${ }^{27,28}$ as well as for patients who are uncomfortable or who develop orthodontic deformities using long term mouthpiece or oral-nasal mask IPPV. ${ }^{27}$ This patient also demonstrated ongoing mucus plugging and severe respiratory difficulties despite tracheostomy. Since his bulbar muscle function was intact prior to tracheostomy, he would have been a candidate for maintenance by strictly noninvasive aids, ${ }^{22,23,29}$ had this been available to him.

Cases 4, 5 and 6 demonstrated inappropriate ventilator weaning. Case 4 was weaned despite symptoms of $\mathrm{CAH}$ and lack of assessment of nocturnal blood gases. Adequate ventilatory assistance eliminated recurrence of pulmonary symptomatology and complications. Case 5 never completely recovered from the respiratory muscle weakness that accompanied his first postinjury URI. ${ }^{30}$ Case 6 was weaned despite grossly inadequate inspiratory muscle function for continued autonomous breathing. This patient demonstrated late plateauing of pulmonary function and safe weaning 7 years postinjury. Although not often recognized, 31 tetraplegics have been reported who regained autonomous breathing a mean of $2.4 \pm 2.2$ years ( 1 month -8 years, median 1.7 years) following onset of injury. ${ }^{29}$

Although, except during URIs, difficulties in clearing airway secretions usually cease following tracheostomy site closure for individuals with intact bulbar musculature, case 7 demonstrated that chronic bronchorrhea could persist. Chronic mucus plugging can hamper ventilator weaning and 
lead to pulmonary complications and repeated bronchoscopy unless eliminated by achieving adequate PCEFs. The unassisted PCEFs in tetraplegic patients usually fail to reach the 5 to $6 \mathrm{l} / \mathrm{s}$ needed for elimination of at least loose airway secretions. ${ }^{25,31}$ Air stacking of ventilator insufflations, or deep insufflations ('sighs') delivered via a mouthor nosepiece can increase PCEFs. ${ }^{25}$ The use of manually assisted coughing, ie timed abdominal thrusts following an inspiration or insufflation greater than $1500 \mathrm{ml}$, can usually raise PCEFs over $5 \mathrm{l} / \mathrm{s} .{ }^{25,31}$ The use of MI-E can create PCEFs of 6 to $10 \mathrm{l} / \mathrm{s}$. ${ }^{25,31}$ The latter is even more effective when used in conjunction with abdominal thrusts during the exsufflation phase. Its use averted further episodes of respiratory failure and bronchoscopy in case 7. Since respiratory muscle dysfunction is increased both during URIs $^{30}$ and by hypercapnia, ${ }^{32.33}$ MI-E was vital in managing the intercurrent URIs for the 4 patients in this study with access to it. Its capacity to eliminate the need for bronchoscopy in ventilator supported individuals has been previously established. ${ }^{34-38}$

Case 8 demonstrated a need for continued evaluation following acute rehabilitation. The need for nocturnal ventilatory assistance is uncommon for adults with greater than $1500 \mathrm{ml}$ or $30 \%-40 \%$ of predicted supine VC unless there are complicating medical conditions, obesity, kyphoscoliosis, malnutrition, or sleep disordered breathing. ${ }^{19}$
In conclusion, these patients demonstrated the late-onset necessity for ventilatory assistance and the effectiveness of regimens of noninvasive IPPV and airway secretion elimination as alternatives to tracheostomy. Any SCI individual with a history of repeated pneumonia, atelectasis, recent ventilatory support requirement, a VC supine less than $30 \%$ to $40 \%$ of predicted normal, nocturnal hypercapnia and mean $\mathrm{SaO}_{2}$ less than $95 \%$, possibly complicating sleep disordered breathing, and possibly subtle symptoms of $\mathrm{CAH}$ is a candidate for nocturnal noninvasive IPPV and the use of expiratory muscle aids as needed. ${ }^{22,23}$ Supplemental oxygen therapy does not decrease the risk of pulmonary complications and is unnecessary for patients without significant intrinsic lung disease who receive adequate ventilatory assistance. It can also hinder the use of oximetry in gauging alveolar ventilation, the effectiveness of noninvasive aids, and the presence and severity of mucus plugging. Patients unable to generate PCEFs of greater than $6 \mathrm{l} / \mathrm{s}$ by manually assisted coughing may benefit from MI-E during periods of airway hypersecretion. Ventilator dependent tetraplegics with adequate bulbar muscle function can also be switched from tracheostomy IPPV to noninvasive IPPV despite having little or no measurable VC or ventilator-free time. The issues concerning this approach have been described. ${ }^{22,29,39}$

\section{References}

1 Boushy SF, Thompson HK Jr, North LB, Beale AR, Snow TR (1973) Prognosis in chronic obstructive pulmonary disease. Am Rev Respir Dis 108: 1373-1383.

2 Inkley SR, Oldenburg FC, Vignos PJ Jr (1974) Pulmonary function in Duchenne muscular dystrophy related to stage of disease. Am J Med 56: 297-306.

3 Bach JR (in press) Update on noninvasive inspiratory and expiratory muscle support: a historical perspective. Chest.

4 Canny GJ, Szeinberg A, Koreska J, Levison H (1989) Hypercapnia in relation to pulmonary function in Duchenne muscular dystrophy. Pediatr Pulmonol 6: 169-171.

5 Braun NMT, Arora NS, Rochester DF (1983) Respiratory muscle and pulmonary function in polymyositis and other proximal myopathies. Thorax 38: 616-623.

6 Smith PEM, Edwards RHT, Calverley PMA (1989) Ventilation and breathing pattern during sleep in Duchenne muscular dystrophy. Chest 96: 1346-1351.

7 Bach JR, Alba AS (1990) Management of chronic alveolar hypoventilation by nasal ventilation. Chest 97 : $52-57$.

8 Bach JR, Alba AS, Saporito LR (1993) Intermittent positive pressure ventilation via the mouth as an alternative to tracheostomy for 257 ventilator users. Chest 103: 174-182.

9 Bach JR, O'Brien J, Krotenberg R, Alba AS (1987) Management of end stage respiratory failure in Duchenne muscular dystrophy. Muscle Nerve 10: 177-182. 
10 Bach JR, McDermott I (1990) Strapless oral-nasal interfaces for positive pressure ventilation. Arch Phys Med Rehabil 71: 908-911.

11 Braun NMT, Marino WD (1984) Effect of daily intermittent rest on respiratory muscles in patients with chronic airflow limitation. Chest 85: 595-605.

12 Cropp A, Dimarco AF (1987) Effects of intermittent negative pressure ventilation on respiratory muscle function in patients with severe chronic obstructive pulmonary disease. Am Rev Respir Dis 135: 1056-1061.

13 Bach JR (in press) A comparison of long-term ventilatory support alternatives from the perspective of the patient and care giver. Chest.

14 Needham CD, Rogan MC, McDonald L (1954) Normal standards for lung volumes, intrapulmonary gas mixing and maximal breathing capacity. Thorax 9: 313-6.

15 Rideau Y, Glorian B, Delaubier A, Tarle O, Bach J (1984) Treatment of scoliosis in Duchenne muscular dystrophy. Muscle Nerve 7: 281-6.

16 Bach J, Alba A, Pilkington LA, Lee M (1981) Long-term rehabilitation in advanced stage of childhood onset, rapidly progressive muscular dystrophy. Arch Phys Med Rehabil 62: 328-331.

17 Fisher AD (1985) Poliomyelitis: late respiratory complications and management. Orthopedics 8: 891-894.

18 Bach JR, Alba AS, Bohatiuk G, Saporito L, Lee M (1987) Mouth intermittent positive pressure ventilation in the management of postpolio respiratory insufficiency. Chest 91: 859-864.

19 Bach JR, Alba AS (1991) Pulmonary dysfunction and sleep disordered breathing as post-polio sequelae: evaluation and management. Orthopedics 14: 1329-1337.

20 Fugl-Meyer AR (1971) Effects of respiratory muscle paralysis in tetraplegic and paraplegic patients. Scand J Rehabil Med 3: 141-150.

21 Mansel JK (1990) Respiratory complications and management of spinal cord injuries. Chest 97: 1446-1452.

22 Bach JR (1991) Alternative methods of ventilatory support for the patient with ventilatory failure due to spinal cord injury. J Am Paraplegia Soc 14: 158-174.

23 Bach JR (1992) Pulmonary rehabilitation considerations for Duchenne muscular dystrophy: the prolongation of life by respiratory muscle aids. Crit Rev Phys Rehabil Med 3: 239-269.

24 Bach JR (1992) Mechanical exsufflation, noninvasive ventilation and new strategies for pulmonary rehabilitation and sleep disordered breathing. Bull N Y Acad Med 68: 321-340.

25 Bach JR, Smith WH, Michaels J, Saporito LS, Alba AS, Dayal R et al (1993) Airway secretion clearance by mechanical exsufflation for post-poliomyelitis ventilator assisted individuals. Arch Phys Med Rehabil 74: 170-177.

26 Bach JR, Penek J (1991) Obstructive sleep apnea complicating negative pressure ventilatory support in patients with chronic paralytic/restrictive ventilatory dysfunction. Chest 99: 1386-1393.

27 Bach JR, Sortor S, Saporito LR (1993) Interfaces for noninvasive intermittent positive pressure ventilatory support in North America. Eur Respir Rev 3: 254-259.

28 McDermott I, Bach JR, Parker C, Sortor S (1989) Custom-fabricated interfaces for intermittent positive pressure ventilation. Int J Prosthodont 2: 224-233.

29 Bach JR, Alba AS (1990) Noninvasive options for ventilatory support of the traumatic high level quadriplegic. Chest 98: 613-619.

30 Mier-Jedrzejowicz A, Brophy C, Green M (1988) Respiratory muscle weakness during upper respiratory tract infections. Am Rev Respir Dis 138: 5-7.

31 Bach JR (in press) Mechanical insufflation-exsufflation: comparison of peak expiratory flows with manually assisted and unassisted coughing techniques. Chest.

32 Rochester DF, Braun NMT (1985) Determinants of maximal inspiratory pressure in chronic obstructive pulmonary disease. Am Rev Respir Dis 132: 42-47.

33 Stubbing DG, Pengelly LD, Morse JLC, Jones NL (1980) Pulmonary mechanics during exercise in subjects with chronic airflow obstruction. J Appl Physiol 49: 511-515.

34 Barach AL, Beck GJ (1954) Exsufflation with negative pressure: physiologic and clinical studies in poliomyelitis, bronchial asthma, pulmonary emphysema and bronchiectasis. Arch Int Med 93: 825-841.

35 Colebatch HJH (1961) Artificial coughing for patients with respiratory paralysis. Australasian J Med 10: 201-212.

36 Bickerman HA (1954) Exsufflation with negative pressure: Elimination of radiopaque material and foreign bodies from bronchi of anesthetized dogs. Arch Int Med 93: 698-704.

37 Williams EK, Holaday DA (1955) The use of exsufflation with negative pressure in postoperative patients. Am J Surg 90: 637-640.

38 Barach AL, Beck GJ, Smith RH (1953) Mechanical production of expiratory flow rates surpassing the capacity of human coughing. Amer J Med Sci 226: 241-248.

39 Bach JR (1991) New approaches in the rehabilitation of the traumatic high level quadriplegic. Am J Phys Med Rehabil 70: 13-20. 